University of Windsor

Scholarship at UWindsor

1974

\title{
Leading radiative correction to the magnetic dipole transition probability
}

Gordon W. F. Drake

University of Windsor

Follow this and additional works at: https://scholar.uwindsor.ca/physicspub

Part of the Physics Commons

\section{Recommended Citation}

Drake, Gordon W. F.. (1974). Leading radiative correction to the magnetic dipole transition probability. Physical Review A, 9 (6), 2799-2801.

https://scholar.uwindsor.ca/physicspub/108

This Article is brought to you for free and open access by the Department of Physics at Scholarship at UWindsor. It has been accepted for inclusion in Physics Publications by an authorized administrator of Scholarship at UWindsor. For more information, please contact scholarship@uwindsor.ca. 


\title{
Comments and Addenda
}

The Comments and Addenda section is for short communications which are not of such urgency as to justify publication in Physical Review Letters and are not appropriate for regular Articles. It includes only the following types of communications: (1) comments on papers previously published in The Physical Review or Physical Review Letters: (2) addenda to papers previously published in The Physical Review or Physical Review Letters. in which the additional information can be presented without the need for writing a complete article. Manuscripts intended for this section should be accompanied by a brief abstract for information-retrieval purposes. Accepted manuscripts will follow the same publication schedule as articles in this journal. and gallevis will be sent to authors.

\section{Leading radiative correction to the magnetic dipole transition probability* \\ G. W. F. Drake ${ }^{\dagger}$ \\ Department of Physics, University of Windsor, Windsor, Ontario, Canada \\ (Received 9 May 1973; revised manuscript received 5 December 1973)}

\begin{abstract}
It has recently been suggested that a radiative correction of relative order $\alpha \ln \alpha$ may contribute to the $1 s 2 s^{3} s_{1}-1 s^{2} S_{0}$ magnetic dipole transition of the heliumlike ions. The low-frequency contributions to the correction are evaluated and shown to vanish.
\end{abstract}

The magnetic dipole transition $1 s 2 s^{3} S_{1}-1 s^{2} S_{0}$ $+h \nu$ of helium and the heliumlike ions is the dominant process in determining the radiative lifetime of the $1 s 2 s^{3} S_{1}$ metastable state. Since the nonrelativistic transition matrix element vanishes, it is necessary to evaluate the relativistic and finite wavelength corrections of relative order $\alpha^{2} Z^{2}$ to obta in a nonvanishing transition probability ${ }^{1-3}$. These terms have been evaluated and decay rates predicted for the heliumlike ions up to $\mathrm{Fe} \mathrm{XXV.}{ }^{1}$ The experimentally measured life times for $\mathrm{HeI},{ }^{4}$ $\mathrm{Cl}$ XVI, ${ }^{5}$ Ar XVII , and Ti XXI, ${ }^{6}$ are all somewhat shorter than the predicted values, suggesting that higher-order radiative corrections may be necessary to bring theory and experiment into agreement. It is tempting to associate the particularly large $30 \%$ discrepancy in the case of heliumlike $\mathrm{Cl} \mathrm{XVI}{ }^{5}$ with the large nuclear magnetic dipole and electric quadrupole moments, but as shown in Ref. 1, these terms are smaller by a factor of $O\left(m_{e} / m_{\text {nuc }}\right)$. Feinberg and Sucher ${ }^{2}$ have suggested that there is a self-interaction radiative correction of order $\alpha \ln \alpha$ relative to the leading nonvanishing term. In this Comment, the low frequency contributions to the $\alpha \ln \alpha$ correction are evaluated and shown to vanish. Other corrections of relative order $\alpha$ and $\alpha^{2} Z^{2}$ are too small to alter significantly the theoretical lifetimes. Some of these effects not involving self-interactions have now been calculated in the Dirac-Hartree-Fock approximation. ${ }^{7}$

The plan of the calculation parallels that for the evaluation of the Lamb shift ${ }^{89}$. Since contributions to the transition moment arising from the electronelectron interaction are smaller than the one-electron terms by at least a factor of $1 / Z$, it is sufficient to consider the corresponding one-electron $2 s-1 s$ transition in hydrogenic ions. The one-electron radiative corrections correspond to the diagrams shown in Fig. 1. As for the Lamb shift, the contributions from low- and high-frequency virtual transverse photons should be evaluated separately. Only the low-frequency contribution is discussed here.

The diagram 1(a) corresponds to the third-order interaction-energy matrix element

$U_{A \rightarrow B}^{(3)}=\frac{2 \alpha}{3 \pi} \int_{0}^{K} k d k \sum_{n, n^{\prime}} \frac{\left(U^{(1)}\right)_{n, n^{\prime}}\left(\psi_{B}, \vec{\alpha} \psi_{n}\right) \cdot\left(\psi_{n^{\prime}}, \vec{\alpha} \psi_{A}\right)}{\left(E_{n}-E_{B}+k\right)\left(E_{n^{\prime}}-E_{A}+k\right)}$,

where $\alpha$ is the fine-structure constant, $k$ is the virtual-photon energy, $K$ is a high-energy cutoff, and $U^{(1)}$ is the first-order interaction-energy operator for a magnetic dipole transition of ener-

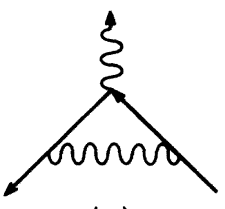

(a)

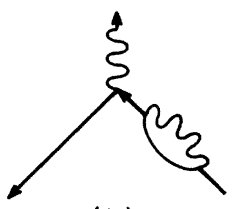

(b)

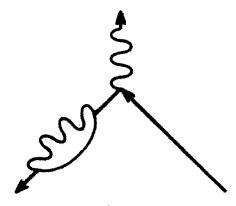

(c)
FIG. 1. One-electron radiative corrections to the spontaneous emission of radiation. 
gy $\hbar \omega=E_{A}-E_{B}$. In the nonrelativistic limit, the $M=0$ component is given by

$$
\begin{aligned}
& U^{(1)}=\left(\frac{2 \hbar \omega}{3 R}\right)^{1 / 2}\left[\frac{\omega}{c}\left(\frac{e}{2 m c} L_{z}+\mu_{z}\right)+O\left(\alpha^{4} Z^{4}\right)\right], \\
& \mu_{z}=(e \hbar / 2 m c) \sigma_{z} .
\end{aligned}
$$

The transition rate is obtained from

$$
\begin{aligned}
& w=\left(2 \pi / \hbar^{2}\right)\left|U_{A \rightarrow B}\right|^{2}(R / \pi c), \\
& U_{A \rightarrow B}=U_{A \rightarrow B}^{(1)}+U_{A \rightarrow B}^{(3)}+\cdots,
\end{aligned}
$$

where $R$ is the radius of a sphere in which the emitted photon wave function of definite angular momentum and parity is normalized, and $U_{A \rightarrow B}^{(1)}$ contains the first-order contributions of $O\left(\alpha^{4} Z^{4}\right)$ evaluated previously, ${ }^{1,2}$ in addition to the terms displayed in Eq. (2). Since the matrix element $\left\langle 1 s\left|U^{(1)}\right| 2 s\right\rangle$ is $O\left(\alpha^{4}\right)$, the corrections to be evaluated are $O\left(\alpha^{5}\right)$ and $O\left(\alpha^{5} \ln \alpha\right)$. In the nonrelativistic limit, $\vec{\alpha}$ in Eq. (1) is replaced by $\vec{v} / c$ and $U^{(1)}$ has only diagonal matrix elements so that

$U_{A \rightarrow B}^{(3)}=\frac{2 \alpha}{3 \pi c^{2}} \int_{0}^{K} k d k \sum_{n} \frac{\left(U^{(1)}\right)_{n, n}\left(\psi_{B}, \overrightarrow{\mathrm{v}} \psi_{n}\right) \cdot\left(\psi_{n}, \overrightarrow{\mathrm{v}} \psi_{A}\right)}{\left(E_{n}-E_{B}+k\right)\left(E_{n}-E_{A}+k\right)}$.

The $L_{z}$ term in Eq. (2) makes no contribution when (5) is summed over intermediate magnetic substates, leaving only the $\mu_{z}$ term.

To the same approximation, the sum of diagrams 1 (b) and 1(c) yields

$$
\begin{aligned}
U_{A \rightarrow B}^{(3)^{\prime}}= & \frac{2 \alpha}{3 \pi c^{2}} \int_{0}^{K} k d k \sum_{n}\left(\psi_{B}, \overrightarrow{\mathrm{v}} \psi_{n}\right) \cdot\left(\psi_{n}, \overrightarrow{\mathrm{v}} \psi_{A}\right) \\
& \times \frac{1}{\hbar \omega}\left(\frac{\left(U^{(1)}\right)_{A, A}}{E_{n}-E_{B}+k}-\frac{\left(U^{(1)}\right)_{B, B}}{E_{n}-E_{A}+k}\right)
\end{aligned}
$$

Since $\left(\mu_{z}\right)_{A, A}=\left(\mu_{z}\right)_{B, B}=\left(\mu_{z}\right)_{n, n}$ for each contributing intermediate state $n$, Eq. (5) and (6) sum to zero in lowest order. In the absence of cancellation, these terms would contribute a correction of $O\left(\alpha^{5} \ln \alpha\right)$.

The analogous calculation for the Lamb shift yields terms containing $\alpha^{5} \ln \left(K / E_{\text {av }}\right)$, while the high-frequency part contributes terms involving $\alpha^{5} \ln (\lambda / m)$, where $\lambda$ is an assumed small photon mass. Use of the relationship $\ln \lambda=\ln 2 K-\frac{5}{6}$ yields a final result independent of both $\lambda$ and $K$, but containing terms of $O\left(\alpha^{5} \ln \alpha\right)$. Since the lowfrequency part of the present calculation is inde- pendent of $K$ to order $\alpha^{5}$, the high-frequency part must be independent of $\ln (\lambda / \mathrm{m})$ to the same order to avoid an infrared divergence in the limit $\lambda \rightarrow 0$. Thus the same terms which give the dominant contribution to the Lamb shift do not contribute to the magnetic dipole transition probability.

A detailed calculation of the high-frequency part, including off-mass-shell and binding-energy effects, is necessary to prove that there are no other sources of $\alpha^{5} \ln \alpha$ terms. Such a calculation is lengthy and intricate, but it seems likely on physical grounds that when all such terms are included, they will sum to zero. For example, the leading term in the anomalous electron magnetic moment is of order $\alpha$ and not $\alpha \ln \alpha$. One would certainly expect an anomalous-magnetic-momenttype correction of $O\left(\alpha^{5}\right)$ from the high-frequency contributions to the magnetic dipole transition. The interaction-energy operator which gives rise to the anomalous magnetic moment of an electron is

$$
\delta U=-(\mu \alpha / 2 \pi)(\beta \vec{\Sigma} \cdot \overrightarrow{\mathcal{H}}-i \beta \vec{\alpha} \cdot \overrightarrow{\mathrm{E}}),
$$

where $\mu=e \hbar / 2 m c, \overrightarrow{\mathcal{H}}=\nabla \times \vec{A}, \vec{E}=-(1 / c) \partial \vec{A} / \partial t$, and $\vec{A}$ is the vector potential of the emitted photon. The equivalent nonrelativistic operator for magnetic dipole transitions obtained as in Ref. 1 by means of a Foldy-Wouthuysen ${ }^{10}$ transformation is

$$
\begin{aligned}
\dot{o} U_{\mathrm{NR}}=(\mu \alpha / 2 \pi) & {\left[\overrightarrow{\boldsymbol{\sigma}} \cdot \overrightarrow{\mathcal{H}}\left(1-\overrightarrow{\mathrm{p}}^{2} / 2 m^{2} c^{2}\right)\right.} \\
& \left.\left.-\overrightarrow{\mathcal{H}} \cdot \overrightarrow{\mathrm{r}} \times(\nabla V \times \vec{\sigma}) / 2 m c^{2}\right)\right]
\end{aligned}
$$

with $V=-Z e^{2} / r$. The above can be written in the form

$$
\delta U=(\alpha / 2 \pi)\left[U^{(1)}-\overrightarrow{\mathcal{F}} \cdot \overrightarrow{\mathrm{p}} \times(\overrightarrow{\mathrm{p}} \times \vec{\sigma}) / 4 m c^{2}\right]
$$

where $U^{(1)}=\vec{Q}_{1} \cdot \overrightarrow{\mathcal{H}}$ and $\vec{Q}_{1}$ is given by Eq. (30) of Ref. 1. For the $2 s-1 s$ transition of hydrogenic ions, the matrix element is

$$
\left\langle 1 s\left|U^{(1)}+\delta U_{\mathrm{NR}}\right| 2 s\right\rangle=(1+\alpha / 4 \pi)\left\langle 1 s\left|U^{(1)}\right| 2 s\right\rangle
$$

since the matrix element of the second term in Eq. (9) is exactly half that of the first. This result is only approximately valid for the $1 s 2 s^{3} S_{1}$ $-1 s^{21} S_{0}$ transition of helium, but is a good approximation for large values of the nuclear charge. There may, of course, be other contributions of $O\left(\alpha^{5}\right)$ arising from the high-frequency part or from virtual transitions to negative frequency states. 
*Research supported by the National Research Council of Canada.

$\dagger$ Alfred P. Sloan Research Fellow.

${ }^{1}$ G. W. F. Drake, Phys. Rev. A 3 , 908 (1971).

${ }^{2}$ G. Feinberg and J. Sucher, Phys. Rev. Lett. 26, 681 (1971).

${ }^{3}$ G. W. F. Drake, Phys. Rev. A 5, 1979 (1972).

${ }^{4}$ H. W. Moos and J. R. Woodworth, Phys. Rev. Lett. $\underline{30}$, 775 (1973).

${ }^{5}$ C. L. Cocke, B. Curnette, and R. Randall, Phys. Rev.
Lett. 31, 507 (1973).

${ }^{6}$ R. Marrus and R. W. Schmieder, Phys. Rev. A $\underline{5}, 1160$ (1972); H. Gould, R. Marrus, and R. W. Schmieder, Phys. Rev. Lett. 31, 504 (1973).

${ }^{7}$ W. R. Johnson and C. -P. Lin, Phys. Rev. A 9, 1489 (1974).

${ }^{8} \mathrm{H}$. A. Bethe, Phys. Rev. 72,339 (1947).

${ }^{9}$ N. M. Kroll and W. E. Lamb, Phys. Rev. 75,388 (1949).

${ }^{10}$ L. L. Foldy and S. A. Wouthuysen, Phys. Rev. 78,29 (1950). 